\title{
Neuroblastic Tumor
}

National Cancer Institute

\section{Source}

National Cancer Institute. Neuroblastic Tumor. NCI Thesaurus. Code C6963.

A group of nervous system tumors which display neuronal differentiation. It includes tumors that are composed of immature round cells and tumors that display advanced differentiation and the formation of gang lion cells. 